\title{
A common SCN5A polymorphism modulates the biophysical effects of an SCN5A mutation
}

\author{
Prakash C. Viswanathan, ${ }^{1}$ D. Woodrow Benson, ${ }^{2}$ and Jeffrey R. Balser ${ }^{1,3}$ \\ ${ }^{1}$ Department of Anesthesiology, Vanderbilt University School of Medicine, Nashville, Tennessee, USA \\ ${ }^{2}$ Children's Hospital Medical Center, Cincinnati, Ohio, USA \\ ${ }^{3}$ Department of Pharmacology, Vanderbilt University School of Medicine, Nashville, Tennessee, USA
}

\begin{abstract}
Our understanding of the genetic basis of disease has expanded with the identification of rare DNA sequence variations ("mutations") that evoke inherited syndromes such as cystic fibrosis, congenital epilepsy, and cardiac arrhythmias. Common sequence variants ("polymorphisms") have also been implicated as risk factors in multiple diseases. Mutations in $S C N 5 A$, the cardiac $\mathrm{Na}^{+}$channel gene, that cause a reduction in $\mathrm{Na}^{+}$current may evoke severe, life-threatening disturbances in cardiac rhythm (i.e., Brugada syndrome), isolated cardiac conduction disease, or combinations of these disorders. Conduction disease is manifest clinically as heart rate slowing (bradycardia), syncope, or "lightheadedness". Recent electrophysiologic studies reveal that mutations in particular families exhibiting cardiac conduction disease cause marked effects on several competing voltage-dependent gating processes, but nonetheless cause a mild "net" reduction in $\mathrm{Na}^{+}$current. Here we show that a common $S C N 5 A$ polymorphism (H558R) in the $\mathrm{Na}^{+}$channel I-II interdomain cytoplasmic linker, present in $20 \%$ of the population, can mitigate the in vitro effects of a nearby mutation (T512I) on $\mathrm{Na}^{+}$channel function. The mutation and the polymorphism were both found in the same allele of a child with isolated conduction disease, suggesting a direct functional association between a polymorphism and a mutation in the same gene.
\end{abstract}

This article was published online in advance of the print edition. The date of publication is available

from the JCI website, http://www.jci.org. J. Clin. Invest. 111:341-346 (2003). doi:10.1172/JCI200316879.

\section{Introduction}

Inherited mutations in the family of genes encoding voltage-gated $\mathrm{Na}^{+}$channel pore-forming subunits (SCN $\underline{X} A)$ underlie a wide spectrum of neurologic, musculoskeletal, and cardiovascular disorders. Mutations in SCN5A, the cardiac $\mathrm{Na}^{+}$channel gene, generally evoke disturbances in cardiac rhythm (1-3); however, it is increasingly clear that a great many single amino acid substitutions within the SCN5A coding region can evoke a broad spectrum of cardiac rhythm behavior. While patients with SCN5A mutations linked to either long QT syndrome or Brugada syndrome experience sudden, life-threatening arrhythmias, patients with isolated conduction disease exhibit heart rate slowing (bradycardia) that manifests clinically as syncope, or perhaps only as lightheadedness.

Functional biophysical studies of cardiac $\mathrm{Na}^{+}$channels with Brugada syndrome mutations invariably reveal defects in either gating function or surface mem-

Received for publication September 10, 2002, and accepted in revised form December 17, 2002.

Address correspondence to: Jeffrey R. Balser, Vanderbilt University School of Medicine, Room 560, Preston Research Building, 2220 Pierce Avenue, Nashville, Tennessee 37232-6602, USA. Phone: (615) 936-0277; Fax: (615) 936-2980;

E-mail: jeff.balser@vanderbilt.edu.

Conflict of interest: The authors have declared that no conflict of interest exists.

Nonstandard abbreviations used: atrioventricular (AV); first depolarized pulse (P1); second depolarized pulse (P2). brane expression that lead to a marked reduction in $\mathrm{Na}^{+}$current (4-6). This causes a disproportionate hastening of repolarization in the epicardial cell layer, leading to a transmural electrical gradient that provokes reentrant arrhythmias $(7,8)$. More recent studies of $\mathrm{Na}^{+}$ channels have identified particular mutations linked to isolated conduction disease; these studies have revealed defects that also lead to a reduction of $\mathrm{Na}^{+}$current, and in some cases the ion channel functional defect is mild $(3,9)$. Whereas even a single $\mathrm{Na}^{+}$channel mutation may cause multiple changes in gating function, each of which could individually drastically increase or decrease the $\mathrm{Na}^{+}$current, computational models of cardiac excitability equipped to consider the ensemble of these mutational effects may predict only a mild net decrease in $\mathrm{Na}^{+}$current (3).

Mutations associated with a reduction of $\mathrm{Na}^{+}$current may evoke Brugada syndrome, conduction disease, or both. While the severity of the $\mathrm{Na}^{+}$channel functional defect may underlie the ECG phenotype in some cases (3), other unrecognized factors (i.e., humoral regulation, auxiliary subunits, transcriptional regulation) are certain to play a role. A recent study identified a new Brugada syndrome locus (10) distinct from SCN5A and associated with progressive conduction disease, suggesting that other genes could also play a role in the manifestation of the disease phenotype.

Most contemporary studies of ion channels have sought to characterize the functional effects of isolated mutations linked to rare diseases. At the same time, 
a I
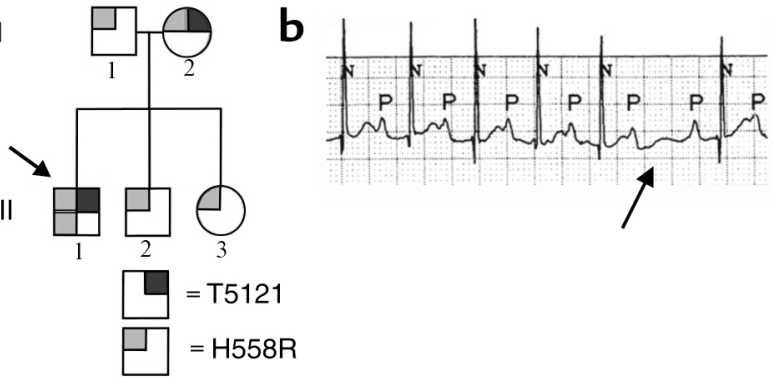

C

T5121

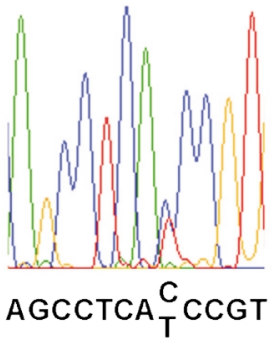

$S \quad L \quad T \quad R$
H558R

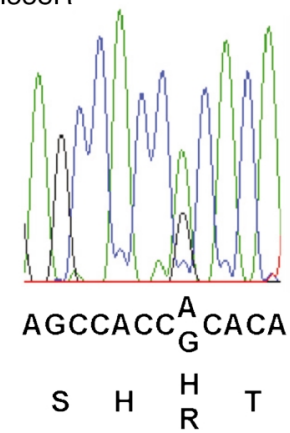

Figure 1

Genotype and ECG phenotype. (a) Pedigree shows affected individuals. Gray boxes represent the polymorphism, H558R, while black boxes represent the T512I mutation. While the father (I-1) was heterozygous for $\mathrm{H} 558 \mathrm{R}$, the mother (I-2) was heterozygous for $\mathrm{H} 558 \mathrm{R}$ and T512I. The proband (arrow, II-1) was homozygous for H558R and heterozygous for T512I while his siblings (II-2 and II-3) were heterozygous for H558R. (b) ECG of proband indicating a second-degree conduction block with normal QT and QRS durations. (c) Sequence analysis of SCN5A reveals a change of threonine to isoleucine at position 512 and a change of histidine to arginine at position 558.

single-nucleotide polymorphisms, DNA sequence variations that are common in the population, have been implicated in phenotypic variability in physiology, pharmacology, and pathophysiology (11-14). Recently, a polymorphism in SCN5A (Y1102) was identified in individuals of African descent and implicated in an elevated risk for arrhythmia (15). Surprisingly, studies have not identified polymorphic alleles that modulate disease phenotypes by influencing the effects of inherited mutations within the same gene. Here we show that a common SCN5A allele (H558R), present in one of five people (16), mitigates the gating defect caused by a nearby mutation (T512I) in the cardiac $\mathrm{Na}^{+}$channel DI-DII linker. The mutation and the polymorphism were both found in the same allele of a child with conduction disease, and are the first to suggest a direct functional interaction between a common polymorphism and a rare mutation within the same gene.

\section{Method}

Mutagenesis. Site-directed mutagenesis (T512I) was performed on SCN5A cDNA cloned in PSP64T as previously described $(17,18) .1795$ ins D mutant and H558R were prepared using the QuikChange Mutagenesis Kit

from Stratagene (La Jolla, California, USA), using hH1/pCGI-WT as template. H558R/T512I and H558R/1795ins D double mutants were made by the same mutagenesis using H558R-hH1/pCGI and $1795 i n s \mathrm{D} / \mathrm{pCGI}$ as template, respectively, for bicistronic expression of the channel protein and GFP reporter in tsa-201 or HEK 293 cells. Cells were cotransfected with an equimolar ratio of $\mathrm{Na}^{+}$channel human $\beta 1$ subunit (provided by Al George, Department of Medicine, Vanderbilt University School of Medicine).

Electrophysiology. All whole-cell $\mathrm{Na}^{+}$currents were recorded at $21^{\circ} \mathrm{C}$ to limit the voltage-clamp error during activation and inactivation gating at higher temperatures. The pipette solution contained $10 \mathrm{mM} \mathrm{NaF}$, $110 \mathrm{mM} \mathrm{CsF}, 20 \mathrm{mM} \mathrm{CsCl}, 10 \mathrm{mM}$ EGTA, and $10 \mathrm{mM}$ HEPES (pH adjusted to 7.35 with $\mathrm{CsOH}$ ); the bath solution contained $145 \mathrm{mM} \mathrm{NaCl}, 4 \mathrm{mM} \mathrm{KCl}, 1.8 \mathrm{mM}$ $\mathrm{CaCl}_{2}, 1 \mathrm{mM} \mathrm{MgCl}_{2}$, and $10 \mathrm{mM}$ HEPES (pH 7.35). Currents were sampled at $20 \mathrm{kHz}$ using a Digidata 1200 analog to digital board (Axon Instruments Inc., Foster City, California, USA) and low-pass filtered at $2 \mathrm{kHz}$. The data were acquired using pClamp 8.0.1 (Axon Instruments Inc.) and analyzed using Clampfit (Axon Instruments Inc.). Voltage-clamp protocols are provided as insets within each figure. The results are expressed as mean $\pm S E M$, and statistical comparisons were made by one-way ANOVA using Origin software (Microcal Software Inc., Northampton, Massachusetts, USA), with $P<0.05$ indicating significance. Multiexponential functions were fitted to the data using the nonlinear least-squares method with Origin software.

\section{Results}

Clinical characteristics and genetic findings. We studied a family who came to medical attention when the proband, a 2-year-old at the time of initial examination (for pedigree see Figure 1a, II-1), was evaluated for an irregular heartbeat. A second-degree atrioventricular (AV) block was detected and treated with a pacemaker. The ECG trace (Figure 1b) shows a background heart rate of approximately 130 beats per minute (normal for a 2-year-old). The QRS duration and QT interval are normal. On conducted beats, the PR interval is relatively stable and prolonged to $200-240 \mathrm{~ms}$ (normal for age 2 years is less than $150 \mathrm{~ms}$ ), a pattern suggestive of type 2 conduction system (Purkinje) block. In addition, the ECG shown (arrow) indicates an intermittent blocked beat, with shortening of the PR interval on the beat immediately following block, a feature suggestive of type $1(\mathrm{AV})$ block. The parents and siblings have a normal ECG (normal QRS, PR, and QT intervals), and there was no evidence of maternal antibody-associated heart block (19).

SCN5A was evaluated as a candidate gene, and mutations were sought using direct, bidirectional sequencing of the coding region. Sequence analysis revealed a change of cytosine to thymine (C1535T), resulting in an amino acid change from threonine to isoleucine (T512I) at codon 512, and a change of adenine to gua- 


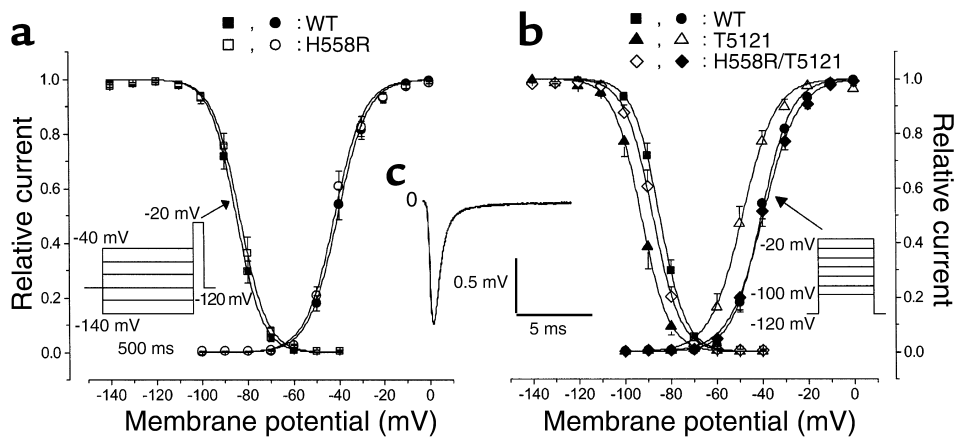

\section{Figure 2}

Steady-state gating parameters. (a) Voltage dependence of activation and inactivation of wild type and H558R obtained using the protocols shown in the inset and fitted to a Boltzmann function. (b) Activation and inactivation parameters of wild type, T512I, and H558R/T512I fitted to a Boltzmann function. Note the hyperpolarizing shifts in activation and inactivation curves as a result of the mutation as well as their restoration by H558R. (c) Wild-type and T512I I Na $_{\text {a }}$ transients obtained during depolarization to $-20 \mathrm{mV}$ from a holding potential of $-120 \mathrm{mV}$ are normalized to illustrate similarity in fast inactivation. nine (A1673G), resulting in an amino acid change from histidine to arginine (H558R) at codon 558 (Figure 1c). The nucleotide change $\mathrm{C} 1535 \mathrm{~T}$ creates a unique BstF5I restriction site, while the nucleotide change A1673G creates an AciI restriction site, allowing independent confirmation of both sequence changes (T512I and H558R). The father was heterozygous for H558R, while the mother was heterozygous for H558R and T512I with both sequence changes on the same allele. The proband (Figure 1a, II-1) was homozygous for H558R and heterozygous for T512I. His siblings (Figure 1a, II-2, II-3) were heterozygous for H558R and did not carry T512I. To verify that the polymorphism and mutation were on the same allele, exon 12 (from the proband) was PCR amplified and subcloned using the TA cloning method (Invitrogen Corp., San Diego, California, USA). The four individual clones were then evaluated by restriction digest with BstF5I and AciI; two clones contained both T512I and H558R (inherited from the mother) and two contained H558R (inherited from the father).

T512I and H558R: competing effects on voltage-dependent gating. We expressed SCN5A wild-type, T512I, and H558R channels in tsa-201 cells to allow whole-cell voltage-clamp measurements. Figure $2 \mathrm{c}$ shows a typical $\mathrm{Na}^{+}$current $\left(\mathrm{I}_{\mathrm{Na}}\right)$ recorded during a step depolarization to $-20 \mathrm{mV}$ from a holding potential of $-120 \mathrm{mV}$. We measured peak $\mathrm{I}_{\mathrm{Na}}$ at varying activating voltages (protocol inset, Figure $2 \mathrm{~b}$ ) and after conditioning steps to a range of inactivating voltages (protocol inset, Figure 2a). The voltage dependence of activation (circles) and inactivation (squares) for wild-type (filled symbols) and H558R (open symbols) channels were plotted (Figure 2a), and voltage-dependent parameters were derived by fitting a Boltzmann function to the data (Table 1). Over a broad range of membrane potentials, activation and inactivation of wild-type and H558R channels were similar. In contrast, the voltage-dependence of activation (open triangles in Figure 2b) and inactivation (filled triangles in Figure $2 \mathrm{~b}$ ) of T512I was shifted negatively by $8-9 \mathrm{mV}$ (Table 1). Since the proband was heterozygous for T512I and homozygous for H558R, we also investigated the gating of the double mutant H558R/T512I. Figure 2b shows activation (filled diamond) and inactivation (open diamond) of the H558R/T512I construct. Surprisingly, H558R eliminated the negative shift in activation and inactivation.

Enhanced inactivation and conduction disease. Given that the polymorphism entirely corrected the T512Iinduced effects on the voltage dependence of gating, we considered whether residual kinetic changes in H558R/T512I could explain the slow conduction phenotype observed. Upon depolarization, $\mathrm{Na}^{+}$channels rapidly open and "fast inactivate" within a few milliseconds, resulting in a transient inward current. To determine whether the rate of fast inactivation gating was modified by the mutation, we compared the decay of wild-type and T512I $\mathrm{I}_{\mathrm{Na}}$ upon step depolarization to test potentials ranging from $-40 \mathrm{mV}$ to $0 \mathrm{mV}$. Figure $2 \mathrm{c}$ shows representative $\mathrm{I}_{\mathrm{Na}}$ recordings from wild-type and T512I channels during a step depolarization from a holding potential of $-120 \mathrm{mV}$ to $-20 \mathrm{mV}$. At all voltages tested, the rate of decay was similar.

Recent studies have demonstrated a pathophysiologic role for slower kinetic components of inactivation that develop throughout the time course of the cardiac action potential and delay recovery from inactivation between heartbeats $(5,6,20)$. In Brugada syndrome, enhanced "slow" inactivation significantly delays the recovery of $\mathrm{Na}^{+}$channels between stimuli, causing a cumulative loss of function, particularly at rapid heart rates due to the shortened diastolic interval (5). We examined slow inactivation of T512I mutants using a dual-pulse protocol to mimic consecutive stimuli
Table 1

Voltage dependence of activation and inactivation

\begin{tabular}{llcccc}
\hline & \multicolumn{2}{c}{ Inactivation } & \multicolumn{2}{c}{ Activation } \\
& & \multicolumn{1}{c}{$V_{1 / 2}$} & $k$ & $V_{1 / 2}$ & $k$ \\
Wild type & $(n=11)$ & $-85.0 \pm 1.2$ & $5.2 \pm 0.2$ & $-40.7 \pm 1.4$ & $5.9 \pm 0.4$ \\
H558R & $(n=10)$ & $-83.2 \pm 1.4$ & $5.0 \pm 0.3$ & $-42.1 \pm 1.3$ & $5.7 \pm 0.5$ \\
T512I & $(n=7)$ & $-92.5 \pm 1.7^{\mathrm{A}}$ & $4.8 \pm 0.3$ & $-49.4 \pm 1.8^{\mathrm{A}}$ & $6.6 \pm 0.3$ \\
H558R/T512I & $(n=10)$ & $-87.2 \pm 1.3^{\mathrm{B}}$ & $5.2 \pm 0.1$ & $-40.0 \pm 1.8^{\mathrm{C}}$ & $6.8 \pm 0.3$
\end{tabular}

Data points shown in Figure 2 were fit using a Boltzmann function of the form $y=[1+$ $\left.\exp \left\{\left(V-V_{1 / 2}\right) / k\right\}\right]^{-1}$, where $V_{1 / 2}$ is the half maximal voltage of activation and $k$ is the slope factor. Activation and inactivation of wild type and H558R were similar, while T512I shifted these parameters negatively by $8-9 \mathrm{mV}$. ${ }^{A} P<0.01$ compared with wild type. H558R eliminated the negative shift in activation and inactivation caused by T512I. ${ }^{\mathrm{B} P}<0.05$ compared with T512I. ${ }^{C} P<0.01$ compared with T512I. 
a
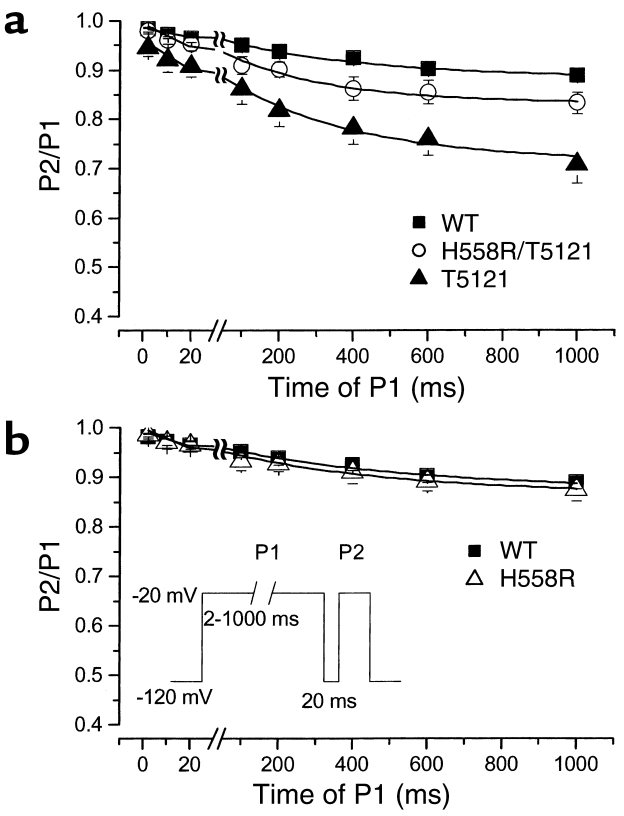

(inset, Figure 3b). As the duration of the first depolarization pulse (P1) was increased, the extent of slow inactivation increased, as indicated by the fractional reduction in peak $\mathrm{I}_{\mathrm{Na}}$ during the $\mathrm{P} 2$ pulse relative to that recorded in the $\mathrm{P} 1$ pulse. A 20 -ms repolarization to $-120 \mathrm{mV}$ was interposed between P1 and P2 to allow recovery from fast, but not slow, inactivation. Figure $3 \mathrm{a}$ shows that with increasing P1 duration, the current elicited by the $\mathrm{P} 2$ pulse decreased due to slow inactivation. For the wild type, the extent of slow inactivation was similar to previously published results (21). In contrast, T512I markedly increased the development of slow inactivation (Figure 3a), whereas H558R did not change the extent to which slow inactivation developed (Figure 3b). Given that H558R appeared to moderate the effect of T512I on the voltage dependence of inactivation gating (Figure 2), we hypothesized that H558R may also limit the extent to which slow inactivation develops with T512I. To test this, we assessed the development of slow inactivation of H558R/T512I (Figure $3 a)$. We found that H558R/T512I significantly enhanced slow inactivation relative to the wild type but to a lesser extent than T512I alone (see Table 2), lending support to our hypothesis.

The detailed kinetics of recovery from inactivation were evaluated using a paired-pulse protocol wherein the hyperpolarizing interval before the second depolarization pulse (P2) was varied (Figure 4a, inset). Both wild-type and mutant data followed a biphasic time course, reflecting components of recovery attributable to fast and slow inactivation. The kinetics of H558R recovery were indistinguishable from wild type (data not shown, but see Table 3). However, recovery of T512I was significantly slower than the wild type (Figure $4 \mathrm{a}$ and Table 3). Once again, the double mutation

\section{Figure 3}

Development of slow inactivation. (a) Slow inactivation was evaluated using the two-pulse protocol shown in the inset in $\mathbf{b}$. Plot shows the ratio of P2/P1 for wild-type, T512I, and H558R/T512I as a function of duration of P1 pulse. Data points were fitted using a two-exponential function. While T512I dramatically enhanced slow inactivation, H558R attenuated slow inactivation caused by T512I alone. (b) Slow inactivation of wild type and H558R. Inset shows the protocol used for evaluation. Slow inactivation of H558R was not different from wild type.

H558R/T512I significantly hastened recovery from inactivation compared with T512I alone $(P<0.05$, Figure $4 \mathrm{a}$ and Table 3 ). These results also point to a modulatory role of the H558R polymorphism on the slow inactivation gating characteristics of the mutation.

To investigate whether H558R modulation of slow inactivation is localized to companion mutations in the DI-DII linker, we engineered the C-terminal mutation 1795ins D and H558R in the same cDNA and expressed them in HEK 293 cells. The 1795 ins D mutation enhances slow inactivation and elicits Brugada syndrome, as reported previously (5) (Figure 4b). H558R had no effect on the kinetic features of 1795ins D (Figure $4 \mathrm{~b}$ ), suggesting that the modulatory role of H558R cannot be generalized to remote channel loci and may be specific to nearby mutations within the DI-DII linker.

\section{Discussion}

Mutations of the human cardiac $\mathrm{Na}^{+}$channel gene (SCN5A) underlie multiple cardiac diseases, including long QT syndrome and Brugada syndrome. While most of the mutations that cause long QT syndrome result in a gain of $\mathrm{Na}^{+}$channel function, mutations that cause Brugada syndrome invariably result in a loss of $\mathrm{Na}^{+}$channel function. Recent studies have identified yet another class of mutations in the $\mathrm{Na}^{+}$ channel that cause isolated conduction disease. Tan et al. (3) provided the first evidence that a mutation in SCN5A causes competing shifts in activation and inactivation gating, where the net effect is a small reduction in $\mathrm{Na}^{+}$current - sufficient to cause conduction slowing, but not Brugada syndrome. While this suggests that manifestation of a particular rhythm phenotype (Brugada syndrome or conduction
Table 2

Development of slow inactivation

\begin{tabular}{lccccc}
\hline & & $A_{1}$ & $A_{2}$ & $\tau_{\text {fast }}(\mathrm{ms})$ & $\tau_{\text {slow }}(\mathrm{ms})$ \\
Wild type & $(n=9)$ & $0.03 \pm 0.005$ & $0.08 \pm 0.007$ & $13 \pm 5$ & $583 \pm 61$ \\
T512I & $(n=7)$ & $0.07 \pm 0.01$ & $0.22 \pm 0.01^{\mathrm{A}}$ & $7 \pm 3$ & $565 \pm 59$ \\
H558R/T512I & $(n=6)$ & $0.04 \pm 0.009$ & $0.12 \pm 0.01^{\mathrm{B}}$ & $10 \pm 1$ & $410 \pm 83$
\end{tabular}

Data points shown in Figure 3a were fit using a two-exponential function of the form

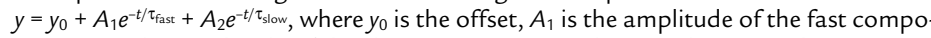
nent, $A_{2}$ is the amplitude of the slow component, and $\tau_{\text {fast }}$ and $\tau_{\text {slow }}$ are the time constants of fast and slow components, respectively. The amplitude $\left(A_{2}\right)$ of the slow component was significantly greater in the mutant than in the wild type $\left({ }^{A} P<0.01\right.$ compared with wild type). However, the time constant $\left(\tau_{\text {slow }}\right)$ was similar to that of the wild type. $\mathrm{H} 558 \mathrm{R}$ attenuated slow inactivation resulting from T512I but was still greater than wild type $\left({ }^{\mathrm{B}} P<0.05\right.$ compared with wild type). 

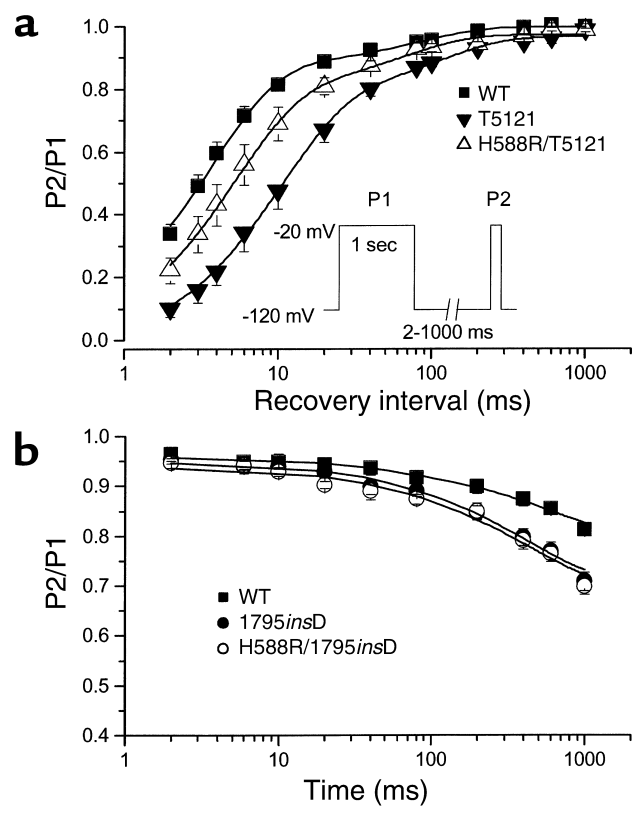

\section{Figure 4}

Recovery from inactivation. (a) Recovery was evaluated using the two-pulse protocol shown in the inset. Data points were fitted using a two-exponential function with the fast time constant corresponding to recovery from fast inactivation and the slow time constant corresponding to recovery from slow inactivation. Plotted are the results obtained from wild type, T512I, and H558R/T512I. (b) Development of slow inactivation of wild type and $1795 \mathrm{ins} \mathrm{D}$ using the protocol shown in Figure $3 \mathrm{~b}$ inset. It is observed that H558R had no effect in attenuating the slow inactivation caused by $1795 \mathrm{ins} \mathrm{D}$.

defect) may depend on the interplay between competing gating lesions, it is also likely that other, unrecognized factors contribute to manifestation of a particular disease phenotype. Recent studies have identified a novel gene locus for Brugada syndrome (10) on chromosome 3 p22-25, distinct from the known $\mathrm{Na}^{+}$ channel loci and overlapping with a previously reported condition arising from locus 3 p22-25 - dilated cardiomyopathy with conduction disease (22). Although the functional role of this gene locus has not been identified, the study suggests that other as yet unidentified genes could play a role in the manifestation of disorders in cardiac excitability.

We identified a novel mutation, T512I, in the $\mathrm{Na}^{+}$channel gene of a 2-year-old boy diagnosed with second-degree $\mathrm{AV}$ conduction block. The T512I mutation, when heterologously expressed, caused hyperpolarizing shifts in activation and inactivation, and also enhanced slow inactivation. However, the common polymorphism H558R also found in this child's $\mathrm{Na}^{+}$ channel gene, which had no effect on wild-type $\mathrm{Na}^{+}$current, attenuated the gating effects caused by mutation T512I. The polymorphism entirely restored the voltage-dependent activation and inactivation voltage shifts caused by the T512I mutation, but only partially restored the kinetic features of slow inactivation. No other family member carried the loss-of-function mutation (T512I) except the mother, who also carried the corrective H558R variant on the same allele (Figure 1a).

While additional studies will be required to firmly establish causality between conduction block and modest changes in slow inactivation, we offer the following hypotheses for linkage between the biophysical observations and the observed phenotypes. The patient described here exhibits AV conduction slowing (prolonged PR intervals) but no intraventricular or intraatrial conduction defect (normal P wave and QRS duration). Recent studies have associated similar $\mathrm{Na}^{+}$ channel slow-inactivation gating abnormalities with $\mathrm{AV}$ conduction defects in patients not exhibiting evidence of other conduction slowing (9). These results contrast markedly with the phenotype of another mutation (G514C) associated with slow AV conduction, as well as delayed conduction throughout the atria and ventricles, including broad P waves, PR interval prolongation, and widening of the QRS complex (3). It is possible that enhanced slow inactivation produced by H558R/T512I, which would cause $\mathrm{Na}^{+}$channels to recover from inactivation more slowly during diastole than wild-type channels do (Figure 3a), provides a mechanism whereby $\mathrm{AV}$ conduction is slowed in preference to atrial or ventricular conduction. Mutations that preferentially delay recovery from inactivation by enhancing slow inactivation could disproportionately affect cells with longer inherent action potential duration (Purkinje cells). In contrast, as in the case of G514C, mutations targeting the channel activation process could affect the myocardium more uniformly, as has been observed (3). Consistent with this idea, it is noteworthy that in the present study H558R entirely eliminated the T512I effect on activation gating (Figure 2b), but only partly corrected the slow inactivation defect. Greater accumulation of $\mathrm{Na}^{+}$channel slow inactivation upon successive stimuli in Purkinje cells, with their longer action potential duration and smaller consequent diastolic interval (23), could lead to greater loss of $\mathrm{Na}^{+}$channel function in these cells at rapid heart rates ( 130 beats per minute, basal heart rate in the proband), and thereby produce isolated AV conduction delay. Moreover, a premature stimulus could

Table 3

Recovery from inactivation

\begin{tabular}{lccccc}
\hline & & $A_{1}$ & $A_{2}$ & $\tau_{\text {fast }}(\mathrm{ms})$ & $\tau_{\text {slow }}(\mathrm{ms})$ \\
Wild type & $(n=7)$ & $0.87 \pm 0.01$ & $0.11 \pm 0.01$ & $3.8 \pm 0.3$ & $85 \pm 7$ \\
H558R & $(n=7)$ & $0.86 \pm 0.03$ & $0.12 \pm 0.03$ & $4.1 \pm 0.4$ & $74 \pm 5$ \\
T512I & $(n=6)$ & $0.82 \pm 0.03$ & $0.16 \pm 0.03$ & $11.6 \pm 1.6^{\mathrm{A}}$ & $137 \pm 14^{\mathrm{A}}$ \\
H558R/T512I & $(n=7)$ & $0.85 \pm 0.01$ & $0.14 \pm 0.01$ & $5.5 \pm 1.1^{\mathrm{B}}$ & $83 \pm 17^{\mathrm{C}}$
\end{tabular}

Data points shown in Figure 4a were fit using a two-exponential function of the form $y=y_{0}+A_{1}\left(1-\exp ^{-t / \tau_{\text {fasc }}}\right)+A_{2}\left(1-\exp ^{-t / \tau_{\text {slow }}}\right)$. While the kinetics of recovery of the wild type and $\mathrm{H} 558 \mathrm{R}$ were similar, $\mathrm{T} 512 \mathrm{I}$ significantly delayed recovery of both components compared with the wild type. ${ }^{A} P<0.01$ compared with wild type. H558R significantly hastened recovery from inactivation resulting from T512I. ${ }^{\mathrm{B} P}<0.01$ compared with $\mathrm{T} 512 \mathrm{I}$. c $P<0.05$ compared with T5121. 
also further compromise the Purkinje diastolic interval and lead to dramatic loss of $\mathrm{Na}^{+}$current and result in all-or-none repolarization and conduction block.

In adulthood, the mother's electrophysiologic phenotype (by history), ECG, and 24-hour ambulatory monitoring was entirely normal. It is possible that the slower heart rate of the mother and consequent longer diastolic interval provides sufficient time for recovery from slow inactivation and thereby did not result in a disease phenotype. While it is unknown whether she displayed ECG abnormalities similar to the proband's during childhood, reduced penetrance of abnormalities in adults with $S C N 5 A$ mutations has been previously described ( 3 , 24). This may also relate to unidentified factors controlling ion channel function and expression.

There is increasing awareness of the role of common polymorphisms in altering gene function and in susceptibility to disease. Studies have linked gene polymorphisms to elevated risk for cystic fibrosis, Alzheimer disease $(12,13)$, and even heart disease $(25,26)$. In addition to their role in disease, polymorphisms are also thought to confer sensitivity to drug therapy (27), as well as proarrhythmic risk from drug therapy $(15,28)$. This study provides the first example in which a polymorphism in the same gene as a rare mutation alters the biophysical effect of the mutation on the channel protein.

\section{Acknowledgments}

This work was supported by the American Heart Association, Southeast Affiliate (to P.C. Viswanathan), and by the NIH (GM-56307 to J.R. Balser, U01 HL-65962 to P.C. Viswanathan, and HD-39946 to D.W. Benson). The authors thank the members of the subject family for their participation. The studies would not have been possible without the technical assistance of Ping $\mathrm{Lu}$ and the assistance of Linda Herrell and Marlene Brabham in collecting patient material.

1. Wang, Q., et al. 1995. SCN5A mutations associated with an inherited cardiac arrhythmia, long QT syndrome. Cell. 80:805-811.

2. Chen, Q., et al. 1998. Genetic basis and molecular mechanism for idiopathic ventricular fibrillation. Nature. 392:293-296.

3. Tan, H.L., et al. 2001. A sodium-channel mutation causes isolated cardiac conduction disease. Nature. 409:1043-1047.

4. Dumaine, R., et al. 1999. Ionic mechanisms responsible for the electrocardiographic phenotype of the Brugada syndrome are temperature dependent. Circ. Res. 85:803-809.
5. Veldkamp, M.W., et al. 2000. Two distinct congenital arrhythmias evoked by a multidysfunctional $\mathrm{Na}(+)$ channel. Circ. Res. 86:E91-E97.

6. Wang, D.W., Makita, N., Kitabatake, A., Balser, J.R., and George, A.L., Jr. 2000. Enhanced $\mathrm{Na}(+)$ channel intermediate inactivation in Brugada syndrome. Circ. Res. 87:E37-E43.

7. Antzelevitch, C., Yan, G.X., and Shimizu, W. 1999. Transmural dispersion of repolarization and arrhythmogenicity: the Brugada syndrome versus the long QT syndrome. J. Electrocardiol. 32(Suppl):158-165.

8. Alings, M., and Wilde, A. 1999. "Brugada" syndrome: clinical data and suggested pathophysiological mechanism. Circulation. 99:666-673.

9. Wang, D.W., Viswanathan, P.C., Balser, J.R., George, A.L., Jr., and Benson, D.W. 2002. Clinical, genetic, and biophysical characterization of SCN5A mutations associated with atrioventricular conduction block. Circulation. 105:341-346.

10. Weiss, R., et al. 2002. Clinical and molecular heterogeneity in the Brugada syndrome: a novel gene locus on chromosome 3. Circulation. 105:707-713.

11. Hull, J., and Thomson, A.H. 1998. Contribution of genetic factors other than CFTR to disease severity in cystic fibrosis. Thorax. 53:1018-1021.

12. Roses, A.D. 1998. Apolipoprotein E and Alzheimer's disease. The tip of the susceptibility iceberg. Ann. NY Acad. Sci. 855:738-743.

13. Saunders, A.M., et al. 2000. The role of apolipoprotein E in Alzheimer's disease: pharmacogenomic target selection. Biochim. Biophys. Acta. 1502:85-94.

14. Wilkins, M.R., Roses, A.D., and Clifford, C.P. 2000. Pharmacogenetics and the treatment of cardiovascular disease. Heart. 84:353-354.

15. Splawski, I., et al. 2002. Variant of SCN5A sodium channel implicated in risk of cardiac arrhythmia. Science. 297:1333-1336.

16. Yang, P., et al. 2002. Allelic variants in long-QT disease genes in patients with drug-associated torsades de pointes. Circulation. 105:1943-1948.

17. Wang, D.W., Yazawa, K., George, A.L., Jr., and Bennett, P.B. 1996. Characterization of human cardiac $\mathrm{Na}+$ channel mutations in the congenital long QT syndrome. Proc. Natl. Acad. Sci. USA. 93:13200-13205.

18. Bezzina, C.R., et al. 1999. A single $\mathrm{Na}(+)$ channel mutation causing both long-QT and Brugada syndromes. Circ. Res. 85:1206-1213.

19. Buyon, J.P. 1998. Autoimmune-associated congenital heart block: demographics, mortality, morbidity and recurrence rates obtained from a national neonatal lupus registry. J. Am. Coll. Cardiol. 31:1658-1666.

20. Rivolta, I., et al. 2001. Inherited Brugada and long QT-3 syndrome mutations of a single residue of the cardiac sodium channel confer distinct channel and clinical phenotypes. J. Biol. Chem. 276:30623-30630.

21. Viswanathan, P.C., et al. 2001. Gating-dependent mechanisms for flecainide action in SCN5A-linked arrhythmia syndromes. Circulation. 104:1200-1205

22. Olson, T.M., and Keating, M.T. 1996. Mapping a cardiomyopathy locus to chromosome 3p22-p25. J. Clin. Invest. 97:528-532.

23. Schram, G., Pourrier, M., Melnyk, P., and Nattel, S. 2002. Differential distribution of cardiac ion channel expression as a basis for regional specialization in electrical function. Circ. Res. 90:939-950.

24. Priori, S.G., Napolitano, C., and Schwartz, P.J. 1999. Low penetrance in the long-QT syndrome: clinical impact. Circulation. 99:529-533.

25. Daley, G.Q., and Cargill, M. 2001. The heart SNPs a beat: polymorphisms in candidate genes for cardiovascular disease. Trends Cardiovasc. Med. 11:60-66.

26. Roses, A.D. 2000. Genetic susceptibility to cardiovascular diseases. Am. Heart J. 140:S45-S47.

27. Roses, A.D. 2000. Pharmacogenetics and the practice of medicine. Nature. 405:857-865.

28. Sesti, F., et al. 2000. A common polymorphism associated with antibiotic-induced cardiac arrhythmia. Proc. Natl. Acad. Sci. USA. 97:10613-10618. 